This is an author-created postprint version. This work has been published in Research in Engineering Design.

The final publication is available at https://doi.org/10.1007/s00163-018-0301-z

\title{
Iterations as the result of social and technical factors: Empirical evidence from a large-scale design project
}

\author{
Sebastiano A. Piccolo*1, Anja M. Maier ${ }^{1}$, Sune Lehmann², and Chris A. McMahon ${ }^{3}$ \\ ${ }^{1}$ Technical University of Denmark, DTU Management Engineering \\ ${ }^{2}$ Technical University of Denmark, DTU Compute \\ ${ }^{3}$ Technical University of Denmark, DTU Mechanical Engineering
}

\begin{abstract}
Understanding the role of iterations is an important topic within design research and design practice. Iterations often involve rework and thus often increased costs and completion time. Many theories and studies ascribe iterations either to the social or technical complexity of the design process. Here, we join the two perspectives by analysing metadata of more than 3000 documents produced during the design of a biomass power plant. We gain insights by using network analysis and by visualising the temporal unfolding of the design process. Subsequently, we develop a statistical model to rigorously test multiple hypotheses showing that iterations are a combination of technical and social factors. The paper shows that iterations increase when the number of stakeholders/participants increases and when external suppliers are involved. Iterations are lower in presence of modular activities. Furthermore, we show the existence of synergistic interactions between nodes' in-going and out-going flow in both activity and team networks associated with an increase in iterations.
\end{abstract}

Keywords: Process Architecture, Organisational Structure, Iterations, Stakeholder, Modularity

\section{Introduction}

Effective project planning and management of engineering design processes is key to timely project execution and system delivery. Understanding and illustrating how the design process evolves over time supports project planning and management. In particular, exploring (and exploiting) the iterative nature of the design process can provide deeper understanding of its temporal evolution.

\footnotetext{
*Corresponding author: sebpi@dtu.dk
}

Thinking in terms of iterations instead of linear sequences can help us plan a process and evaluate how changes will impact cost; for instance by using simulations with explicit probabilities of rework 8]. Whilst simulations are useful and enable the creation of what-if scenarios, we argue in this paper that learning from experience by analysing data traces from previous design processes is as important. By focusing on the iterative nature of the design process 44.77 and the many parties involved 9, 10, such analyses can provide strategic insights to better plan future design processes and to obtain information for use in bespoke simulations. Furthermore, the value of data traces to reconstruct temporal dynamics of processes and understanding iterations has been demonstrated 1, 32, 56. Finally, it is important for the goal of creating and studying realistic models that models are based on real observations or that empirical studies support insights from models.

In this paper, we analyse empirical evidence of iterations on a refined scale of single documents for more than 3000 documents produced during the design and development of a biomass power plant. Employing exploratory data analysis, network analysis, and statistical modelling, we show how iterations are the result of combinations of social and technical factors. Doing this, we create a bridge between the social perspective on the design process $[9]$ and the technical perspective $6,23,34$. To create this bridge, we intersect design theory about modularity and iterations with network science to model process structure and organisational structure.

To study the effect of the network structures on the revision of documents, we model the process structure as a directed activity network and the social structure as a directed network of teams. In each network and for each node, we quantify the influence exerted by a node on another node and also dependencies. We show that influence and dependence are in a synergistic relationship that is positively asso- 
ciated with the number of iterations received by a document. That is, the more an activity can influence other activities and, at the same time, the more an activity depends on other activities, the higher the number of iterations of the documents connected to it. Similar reasoning applies to teams: the more a team can influence the other teams and the more it depends on others, the higher the number of revisions exhibited by the documents that the team produces.

Exploiting the connection between activities and product subsystems, we confirm the beneficial effects of modularity as a design strategy to reduce or mitigate iterations. We find that documents associated with modular subsystem activities, that is activities that map to modular subsystems [53], receive $30 \%$ fewer revisions than documents associated with integrative subsystem activities. Furthermore, we refine previous insights about the negative relation between performance and number of participants 9 , 47, showing that iterations increase as the number of stakeholders/project participants with decision power increases and when external partners are involved.

With this paper, we contribute to the stream of literature that develops the socio-technical understanding of engineering design and product development [11, 17, 29, 57, 65, by considering process, organisational, and product (through the mapping of activities to subsystems) structures. As the process structure is available in the planning stage, our analysis helps project planners and managers to detect activities to monitor and to rethink the process structure to lower the risk of iterations. In addition, with this work, the communication structure between the teams can be retrieved and the teams' influence and dependence can be estimated. This provides information on how to structure collaboration, indicates where to intervene, and how to lighten the responsibilities of the teams for better information processing capacity. Owing to the availability of the data sources, our methodology can be leveraged in both planning and execution stages.

After setting the stage by reviewing the fundamentals of design processes and iterations and studies that use documents or their metadata to understand the design process in $\$ 2$, we derive a theoretical framework and testable hypotheses from previous literature in $\$ 3$ to test the social and technical factors described above. In $\$ 4$, we present our case and dataset and in $\$ 5$ we conduct an exploratory analysis to visualise the design process, understand it, refine two hypotheses related to document usage, and implement the network centrality to successively test relations between organisational structure and iterations. In $\$ 6$, \$7 and $\$ 8$ we test our hypotheses using a multiple regression framework, and in $\$ 9$ and $\$ 10$ we discuss the implications of our results for researchers and practitioners, envisaging future directions of research.

\section{Background and related litera- ture}

The design process is a social process involving many parties and interactions between them to reach a commonly accepted design [9]. Designers externalise their thoughts and communicate using different representations with different purposes [31]. Designers generate ideas and place their thoughts in documents and, as their ideas evolve, the documents they created evolve accordingly $[30]$. Thus, designers can further specialise their documents or change them radically according to their ideas and the social interactions that take place during the design process [31]. These interactions imply changes in the designers' work, potentially adding uncertainty to it, requiring exploration of many alternatives, and making the design process iterative.

Design scholars and practitioners recognise the importance of iterations in design, see, for example, a recent review by Wynn and Eckert 77 and survey results with design experts on iteration as one of the most important characteristics of engineering design processes by Maier and Störrle [44]. Whilst there is agreement on the importance, one may argue that scholars and practitioners have 'mixed feelings' when it comes to the implications of iterations. On the one hand, iterations have positive effects including design progression and on the other hand, iterations are reported to increase duration and cost of a project [77. Wynn and Eckert 77 review perspectives on iterations in design and propose an integrative taxonomy, categorising iteration stereotypes. They propose three general categories: iterations toward progression, iterations for corrections, and iterations for coordination [77. Progressive iterations add value to the project and contribute to refine the specifications, the solution, add functionalities, and, in general, obtain a better design. Corrective iterations are often the response to unplanned adverse events and are perceived as undesirable as they can require new work, rework, and can produce cascade effects (e.g. a solution to a problem generates other problems). Finally, coordinative iterations help to make the process more effective or efficient 77 .

Many scholars embraced the study of the design process to understand iterations from a modelling perspective. These studies develop techniques, mostly based on Design Structure Matrices [23], to optimise execution time [8, 23, to minimise unnecessary iterations [8, 23, to modularise the process 23], etc. Important insights come from simulation approaches: for instance, Braha and Bar-Yam [6] simulated an error propagation process on top of four activity networks observing that activity networks are error tolerant if errors happen at random, but vulnerable if errors happen at central activities, with the consequence of increased exe- 
This is an author-created postprint version. This work has been published in Research in Engineering Design.

The final publication is available at https://doi.org/10.1007/s00163-018-0301-z

cution times. Hoedemaker et al. [34] explored the relationship between the number of modules and the time needed to complete a process, simulating the time needed for coordination, rework, and integration as the number of modules increases, finding that a too high number of modules is counter-productive because the likelihood of iterations occurring during integration stages is higher, limiting the gain obtainable from concurrency.

Other studies take a descriptive approach: they start from data or documents produced during a design process and consider how data or documents evolve over time. A temporal description of the design process can lead to new knowledge on iterations and the way designers work. Techniques like Linkography [30] were used to analyse how designers generate ideas and how they come back to previously generated ideas during the evolution of the design. Goldschmidt [30] shows how the idea generation process follows a logical progression over time. Process mining techniques [1] take a post-mortem perspective on the process: through log-data, they discover a process model (often represented as a Petri Net with focus on activities) aiming to find bottlenecks. The discovered process model can be used for comparison with the planned process model or with new ones, or for simulation purposes. Among descriptive approaches, the analysis of the evolution of digital objects, such as emails, documents created during the design process, and other data traces has been proven relevant to show patterns associated with project milestones 32 . Complementary to [32], we analyse traces of a design process using a document-log. Our results are in line with 32 in that we confirm the importance of metadata for process re-construction. With this paper, however, we go further in that we consider documents, people, and design activities. We use these metadata to test multiple hypotheses about iterations that we derived from previous literature and from our exploratory data analysis. As a first in design literature, we show how metadata reflects the iterativeness of the design process and how the analysis of metadata can be leveraged to obtain a deeper understanding of the design process for both researchers and practitioners.

\section{Theory and hypotheses}

The theoretical framework in this paper is built by connecting the understanding of the design process as a social process of negotiations between many participants with the understanding of the design process as a technical process that involves activities and information dependencies between them (Figure 1). We accomplish this by intersecting network theory and design theory on iterations and modularity, accounting for both the social (organisational) and the technical process network (interdependence between ac-

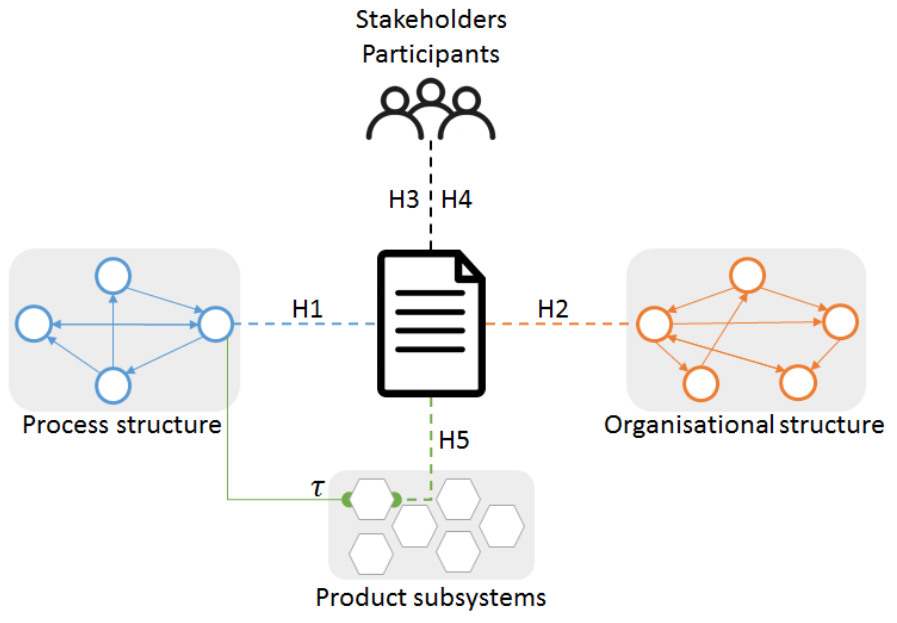

Figure 1: Depiction of the theoretical framework: the document is the unit of analysis and its number of revisions, as proxy of iterations, the phenomenon of interest. We hypothesise that the number of revisions is influenced by the structure of the process (H1), the social structure of teams (H2), stakeholders and participants not directly involved in the document production (H3), external partners (H4), and modulatity or integrativity of product subsystems (H5) that are modelled by mapping activities to subsystems $(\tau)$.

tivities). We connect the social and the technical processes taking place in a design project by using the documents produced during the process as the unit of analysis. As the documents created in the design process go through design reviews 21,35, we use the number of revisions each document receives as a proxy for iterations, as it captures the number of formal iterations for each document.

Each document is elaborated by a team in the context of an activity and during reviewed by other teams. The document production process can be viewed as a directed network of teams, representing the communication structure as the flow of documents among teams 31,56. The flow of documents between teams can affect the number of revisions a document receives. Reviews can highlight problems with documents that need to be addressed, thus adding revisions. In such a network, a team exerts a certain influence on other teams relative to the amount of documents received (for review) from other teams and also depends on other teams relative to the amount of documents that are reviewed by other teams (more details are provided in $\$ 5.3$. Similarly, activities are interconnected by information dependencies $6,23,24$. In fact, an activity can provide outputs to other activities and use the outputs provided by other activities. A change in one activity might propagate to other activities producing other changes $6,14,28$. As 
This is an author-created postprint version. This work has been published in Research in Engineering Design.

The final publication is available at https://doi.org/10.1007/s00163-018-0301-z

such, an activity depends on other activities in proportion to the amount of information provided in input by other activities and exerts an influence on other activities in proportion to the amount of information provided in output. As a document is connected to an activity and to a team, its revisions can be influenced by the properties of both activity and social networks (Figure $1 \mathrm{H} 1$ and H2). We formulate hypotheses on the relation between the structure of information flows, through nodes' centrality of both activity and social networks, and iterations on a document level in $\$ 3.1$ and $\$ 3.2$

Activity and social networks are not the only factors affecting iterations; there are other stakeholders and project participants with decision power that can affect document iterations, such as external companies or the client 9.10 (Figure 1 H3 and H4). We elaborate the hypotheses about stakeholders and project participants in $\$ 3.3$. In addition, the activity network can be substantially different from the product structure 23, thus being insufficient to capture modularity at a product subsystem level. In fact, each activity can be modular or integrative to the extent to which it maps to a modular or integrative subsystem [53. (Figure $1 \tau)$. As modularity has been shown to have positive effects on component quality in terms of smaller number of defects 63, 66, 67, 72 and fewer number of components affected by change propagation [41,42, documents associated with modular subsystems might have lower probability of originating or being affected by changes than documents associated with integrative activities (Figure 11 H5). We detail this hypothesis in 83.4 Finally, we complement our hypotheses with a consideration of the correlation between iterations and execution time in 3.5 .

\subsection{Network theory and iterations: the in- fluence of process structure}

Network theory has been used to establish the role of the most central nodes to spread and/or receive signals circulating in the network $6,54,55,57$. Nodes with high spreading power can propagate signals to other nodes, while nodes with high receiving power are likely to receive many signals. Spreading and receiving power are functions, respectively, of the number of outgoing connections (i.e. out-degree) and the number of incoming connections (i.e. in-degree) that a node has. Some nodes can show high prominence as both spreaders and receivers, and are therefore highly important as they can both facilitate and hinder the circulation of signals in the network 6, 55, 57.

In an activity network, an error in an activity with high spreading power can affect other activities, while an activity with high receiving power is likely to be affected by errors happening in some other activities. As such, given an activ- ity, its spreading power represents the influence the activity exerts on other activities, while its receiving power represents the extent to which the activity depends on other activities. The spreading power is already used in the DSM literature as a means to sequence the activities reducing the expected number of iterations by executing the ones with high spreading power first 23, 24, 68. In fact, errors can cascade through many activities, even from distant areas of the network, with the effect that the affected activities need to be re-iterated $6,28,57,61$. These iterations can manifest in terms of revisions in the documents associated with the affected activities. The situation can be worse in case of an activity exhibiting both high spreading power and high receiving power as the propagation of changes can return back to the originating activity, exacerbating the number of revisions suffered by the documents connected to such activity. Hence, we posit the following hypothesis:

H1: The number of revisions exhibited by a document increases with both the spreading power and the receiving power of the activity with which the document is associated.

We do not refer only to the simple addition between two positive quantities; we want to test also a multiplicative interaction. That is, if we measure the spreading power using out-degree as a proxy and the receiving power using the indegree, this implies that the product between the out-degree and the in-degree will be positively correlated with the number of iterations. Previous research suggested a quadratic, inverted- $\mathcal{U}$, relationship between centrality of components and quality (captured by the number of warranty claims on the component) 29]. Thus, we will also test for an eventual inverted $\mathcal{U}$ relation and, at the same time, to control that an eventual interaction term is not confounded with the curvature defined by the quadratic curve 1927 .

\subsection{Network theory and iterations: the in- fluence of organisational structure}

Similar reasoning can also be made for networks involving humans, where nodes represent people or teams. In the document editing process, teams produce documents that can be reviewed by other teams and vice versa. In such networks, the outgoing flow of a team represents the flow of documents reviewed by other teams, while the ingoing flow represents the flow of documents reviewed for other teams. By producing documents, a team takes a role that we call facilitator (see $\$ 5.3$ because the documents produced might be necessary to other teams to complete their work; however, these documents are subjected to the review of other teams. By reviewing documents, a team takes a role that we call authority (see $\$ 5.3$ because reviewing documents 
This is an author-created postprint version. This work has been published in Research in Engineering Design.

The final publication is available at https://doi.org/10.1007/s00163-018-0301-z

ensures the correctness of the information contained in the document. Therefore, similarly with the dynamics taking place on the activity network, previously discussed, the role of facilitator represents the dependence of a team on other teams and the role of authority represents the influence that a team exerts on the others. A document "rejected" during the review will need changes that can propagate to other documents produced by the same or other teams. Things can be exacerbated for teams that show high prominence in both the role of autority and facilitator. In fact, they can easily be overwhelmed by the amount of information they process, as there are limits to the information processing capacity $48,59,60$. This high flow of documents can be detrimental to performance and thus induce more revisions. Therefore, we posit the following hypothesis:

H2: The number of revisions exhibited by a documents increases with both the prominence as authority and the prominence as facilitator of the team that produces the document.

We measure the prominence of each team to be an authority or a facilitator in the network by using two centrality measures that can account for both the position in the network and the amount of documents processed by each team (see 5.3 . The centrality measures that we use in this case, differently from the degree, do not only account for directed connections but also for indirect ones. As for H1, H2 involves the product of two terms, and we will for the same reasons also here account for the quadratic terms.

\subsection{Impact of stakeholders and project par- ticipants with decision power}

The term stakeholder is often broadly defined as someone who holds a stake or interest in the project [15] and as someone who is affected or can affect the project 26]. This definition is congruent with the definition given by Bucciarelli of participant in a design process [10]: everyone who has a legitimate say in the process, whose actions matter, is a participant. In this regard, stakeholders or participants include: project members, clients, suppliers, regulators and so on $10,15,50$. It can be argued that project participants are also stakeholders but not all stakeholders are project participants (for instance, future generations). For our purposes, hereon we will use the two terms synonymously. It is generally agreed that mismanagement of stakeholders can severely harm the project $15,37,76$. However, stakeholders' participation in the project may also have positive effects. For example, the participation of specific stakeholders has been found to increase the quality of ecosystem plans 7 . The presence of many participants that can influence design processes makes the process inherently social. In such processes, as Bucciarelli argues [9], there is no one dictating the design of an artefact. The artefact itself represents the outcome of many negotiations and thus, potentially, iterations between the participants. Reaching consensus between participants is hard, as they may have conflicting goals $[51$. 73 . Mihm et al. 46 47 have shown through simulations that the number of people in groups is negatively associated with the performance and thus concluded that groups should be kept small. Here, we strive to empirically validate and expand this result including also participants or stakeholders that might not be part of any design team but might have decision power, such as the client. Thus, we posit the following hypothesis:

H3: The number of revisions exhibited by a document increases with its number of stakeholders.

For similar reasons, considering that coordination outside the company's boundaries could be more complicated, that external companies or suppliers might be busy with many other clients, thus being subjected to the limits to information processing capacity, those documents that involve external partners might exhibit more revisions than documents produced internally at the organisation, without involvement of external partners, independently of the number of stakeholders. Thus we posit the following hypothesis:

H4: The number of revisions exhibited by a document, independently of the number of stakeholders, is higher if the document involves external partners.

\subsection{Modularity and iterations}

Sosa et al. 64 66] define modular systems as those systems whose frequency of interfaces within the systems' boundaries exceeds the frequency of interfaces outside the systems' boundaries (according to the $\chi^{2}$ test). Conversely, integrative systems are those systems whose frequency of interfaces outside the system's boundaries exceeds or is comparable to the frequency of interfaces within the system's boundaries. Similar reasoning can be applied to define modular and integrative activities [53 in the context of design processes: modular activities are those activities related to modular product subsystems, while integrative activities are those activities related to integrative product subsystems. Here, we use this definition to account, partially, for the structure of the product. In fact, an activity can map to a modular or integrative subsystem 53. Indeed, it has been reported how the structure of the product influences the amount of rework or defects. Modular components are associated with lower amount of defects and rework than integrative components 14, 17, 22, 41, 42,63,67,72 This was found to be true 
This is an author-created postprint version. This work has been published in Research in Engineering Design.

The final publication is available at https://doi.org/10.1007/s00163-018-0301-z

in different domains: integrated circuit design [70, software engineering [41 43, 63, 67, the construction sector [71], and multiple manufacturing plants operating in mechanical, electronic and transportation equipment sectors [18].

As such, documents associated with modular subsystems, independently of the spreading power and receiving power of the activity they are associated with (see $\$ 3.1$, can have lower probability of being subjected to revisions or changes propagating from other teams, activities, or subsystems. This might happen because modular components have fewer interfaces, thus lower amount of paths available for propagation of changes [14,64, 66, the sets of requirements for modular subsystems are easier to manage and the interfaces between subsystems might have been already defined and fixed 58,60 . Thus, we posit the following hypothesis:

H5: The number of revisions exhibited by a document, independently of the spreading power and receiving power of the activity with which it is associated, increases if the document is associated with an integrative subsystem activity.

\subsection{Completion time and iterations}

A large portion of engineering design literature associates iterations with increase in development time and costs. Lack of adequate information to perform an activity or mistakes committed while executing them can lead to rework 40 69. Some iterations can also lead to more rework by opening more problems while fixing others. For example, Yassine et al. 78 observed churn iterations, where the completion time increases while the work done does not add too much to the design 23. Ford and Sterman 25] observe that if rework takes place under schedule pressure, it can cause even more rework. This rework can increase the completion time and thus costs $2,6,39,62$. Furthermore, it has been shown that errors occurring at highly connected activities, are likely to increase completion time 6]. To avoid these unpleasant scenarios, methods to schedule activities to lower the number of iterations and thus to reduce the overall development time have been proposed [8, $12,13,38,45,68]$. However, it seems that there is no universal relationship between iterations and completion time [77. Indeed, in certain development methodologies such as agile methods, iterations are embraced as a strategy to reduce development time [33. The rationale behind these methodologies is that incremental development driven by many short time iterations helps to reach consensus between participants faster, reducing the total development time 16,33 .

Considering all the above, and the fact that the design process we analyse resembles more a systems engineering approach 74 rather than following an agile development methodology, we posit the following hypothesis:
H6: The number of revisions exhibited by a document is positively correlated with its total editing time.

\section{Case study background}

The dataset we use to investigate the previous hypotheses comes from a multi-project international company developing biomass power plants. The dataset contains a log of the 3559 documents produced during the design process of such a plant for electrical power generation. The process spanned a period of almost four years (2009-2013), with some documents being issued up to two years after project completion. The design process involved more than 100 people and $\sim 150$ design activities. For each document produced, we use the creation date, the completion date, the last transmission date, and the first issuing date. We also find information about the creator, the final modifier, the document title, the document type (drawing, meeting minutes, functional specification, etc.), the number of pages, and the number of revisions. In addition, the log indicates whether the document was sent to the client, to site erection/construction, to quality assessment, or to the plant quality management. Finally, we use the activity code the document is associated with. The document log does not contain the content of the document, nor does it contain the full history of the documents.

The document $\log$ was generated automatically by the company's data management systems and we noted that the log files had some missing data in the form of missing dates. For the exploratory data analysis $(\$ 5)$, we decided to remove all observations where both creation date and completion date were missing at the same time (303 records). The final dataset contains 3256 observations, which represent $92 \%$ of the original data. After data cleaning, we used the documentation obtained to map each person to their functional unit and each activity to their activity group. With functional unit we mean a formal group of people, i.e. a team, in the company organised according to the function they work on and develop in the product (the biomass powerplant). Our case company adopts a functional structure to organise the work rather than a 'product' structure. In the following, we will use the terms functional unit and team interchangeably. To store documents, the company uses a taxonomy indicating the document type in combination with the indications of the activity and the functional unit the document is associated with. Labelling and categorisation was done by the case company. By company policy, each document is associated with only one activity, one functional unit, and one type. Once a document is created, the engineer uploads it to the data management and documentation system, provid- 
This is an author-created postprint version. This work has been published in Research in Engineering Design.

The final publication is available at https://doi.org/10.1007/s00163-018-0301-z

Table 1: Distribution of documents for activity groups (a), for functional units (b), and for document types (c).

(a)

\begin{tabular}{lr}
\hline Activity group & \# docs \\
\hline Air and flue gas & 372 \\
Boiler design & 174 \\
Combustion system & 750 \\
Electrical control & 112 \\
External piping & 534 \\
Others & 40 \\
Project management & 189 \\
PFD + P\&ID & 210 \\
Pressure parts design & 590 \\
Steel related activities & 285 \\
\hline
\end{tabular}

(b)

\begin{tabular}{lr}
\hline Functional unit & \# docs \\
\hline Boiler design & 26 \\
Combustion system & 729 \\
Electrical design & 177 \\
Mechanical design & 177 \\
External consultants & 47 \\
Plant design & 304 \\
Pressure parts design & 1648 \\
Project management & 93 \\
Others & 55 \\
\hline
\end{tabular}

(c)

\begin{tabular}{lr}
\hline Document type & \# docs \\
\hline Drawings & 1368 \\
Foreign documents & 153 \\
Foreign drawings & 866 \\
Meeting minutes & 119 \\
Others & 215 \\
Part lists & 174 \\
Sketches & 138 \\
Technical calculations & 52 \\
Technical documentation & 74 \\
Technical requirements & 97 \\
\hline
\end{tabular}

ing all the information needed for the classification. Table 1 summarises the dataset. We aggregated all categories for which there were less than 25 documents under the label 'Others'. In addition, as the company used some external consultants for short periods of time, we included them under the label 'External consultants'.

\section{Exploratory data analysis}

We perform a broad exploratory analysis of the case dataset with three objectives in mind. First, to describe patterns of iterations during the design process as manifest through actions performed on documents. Second, to trace the temporal evolution of the design process through the documents generated during its progression. Third, to understand communication between people using documents as carriers of information.

\subsection{Global patterns of actions performed on documents: Showing high-level it- erations}

We begin our analysis by making sense of the different actions performed on documents (creation, last modification understood as completion, last transmission, and first issuing). We aggregate the volume of actions performed on documents for each month and compute their relative proportion. Figure 2 shows the result of this analysis in the form of a temporal heat map, where each cell represents a month of work with documents. As we have four types of actions performed on documents, we have, for each month, four cells showing the proportion of each action in that specific month. The darker the colour in the figure, the higher the proportion.
Figure 2 shows which actions are more predominant and when in the process they occur. The data traces left show us the following: we observe a phase from July 2010 to September 2011 with sparse patterns of activities; what we here term a 'negotiation period'. We know that the project was intentionally put on hold due to contracting and other reasons. This time-period appears to be mostly focused on one action, i.e. we find only issuing actions for July-August 2010. After that, we observe a period of no activities until January 2011. In this period, it is likely that the company was waiting for an answer from the client. From February to September 2011, we observe an alternation between first issues, document creations, document completions, and the first completion through the full cycle from first to last transmission logged. This sparse pattern marks the interactions with the client and the consequent iterations and changes to the design process.

We can relate this sequence of actions performed on documents and the completion of the first iteration cycles to what in the literature has been termed 'coordination iterations'. Indeed, in this phase, the company iterates with the client to address the client's requests and to establish some baselines in order to proceed with detailed design. The first documents issued mark such a baseline or inflection point. Furthermore, this 'negotiation period' shows the uncertainty that surrounds the design process: if the client had not signed the contract, the company would have wasted two years of work. This is an example where the blank space may be more telling and explicit than the non-white space. From October 2011 onward, we see that the creation and completion of documents show patterns of complementarity, with periods focused more on new creations than completion and vice versa. From August 2012 until the end of the design process, we observe an increase of last transmissions, 


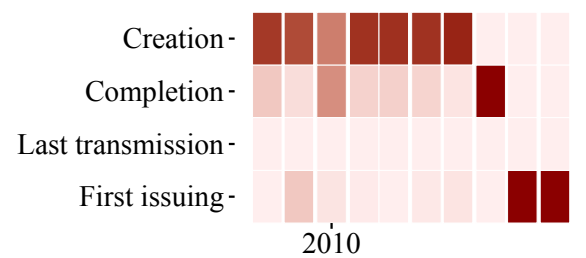

2010
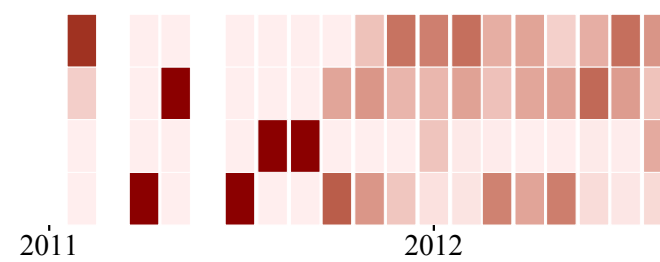

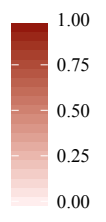

2013

Figure 2: The figure shows the proportion of documents created, completed, transmitted for the last time, and issued for the first time for each month. The map is read by columns and a column represents one month. White spaces represent no data-logging.

which means that people are completing the last coordination iterations. Concordantly, from January 2013 until the end of the design process, we also observe that the focus switches from the creation of documents to their completion. What we see is that until August 2010, up to $12 \%$ of the total documents are created and only a small percentage of these are completed and issued. Then, after the iterations with the client and the approval of the project, we can see a rise in all types of actions (January-February 2012). By March 2012, people had created 25\% of the documents and completed around $12 \%$ of them. Seventy five percent of documents issued received their first issuing by May 2012. Creation and completion show similar progressions, but also patterns of complementarity: an increase in the creation rate often corresponds with a decrease in the completion rate and vice versa. The evolution of the last transmission date shows that roughly $75 \%$ of the coordination iteration cycles are completed in the last 12 months, which is equal to $25 \%$ of the total time. We observe also that documents created towards the end of the design process are completed faster than previous documents. This can indicate that documents created later on in the design process tend to get a smaller amount of revisions. This may happen because the process becomes more mature as the time elapses. Thus, in our model, to explain the number of iterations, we will use the creation date as a control variable.

\subsection{Zooming in on the type of documents: Showing temporal evolution of itera- tions and 'phase transition' in the de- sign process}

Whilst 5.1 provides an overview of the global unfolding of the design process over time, we will now dig deeper into the story that the metadata of documents can tell us. We aim to understand what happened during this design process as it progressed. In particular, we seek to understand which documents people worked on and what happened during the 'negotiation period'. As the majority of documents are completed within one month of their creation, we can compare the proportion of documents created and completed each month using, again, a temporal heat map (Figure 3). From November 2009 to May 2010, we see that people created mostly drawings, with some technical requirements, technical calculations and electrical documents. During this period, people did not use foreign drawings; we see only very few foreign drawings created and none completed. 'Foreign drawings' is a term used by the case company to denote drawings involving also people from outside the focal company, e.g. suppliers.

This is consistent with and deepens further the results found in 5.1 . In the early stages of this design process, it was important to communicate and iterate with the client. Once the client's iterations are addressed, the company can use foreign drawings (company terminology) to communicate and iterate with external providers. In the same period, people completed only a small fraction of drawings, focusing on sketches, electrical documents, and technical calculations. The usage of those types of documents at this stage suggests an incremental development approach. These iterations in the early stages of a design process may suggest exploration activity of the solution space.

During the 'negotiation period', people completed some technical calculations they created previously (June 2010 and May 2011). This is a sign of reworking or incremental iterations: people work on previous documents to include more specifics or to correct issues. In October 2011, people worked on and completed previous technical requirements, without creating new ones. This is also a pattern of rework, but it can also mean that, during negotiations, the company and the client agreed to change previous requirements. In November 2011, we see the creation of new functional specifications in the category 'Others' and rework on technical requirements and technical calculations. The 'negotiation period' is now clearer: the work of the company addressed issues in technical requirements, calculations, functional specifications, and created some new drawings. The intermittent work over time can indicate the necessary time to take decisions and receive approvals. Thus, there is probably more behind-the-stage work, e.g. from sales people, marketing, 
This is an author-created postprint version. This work has been published in Research in Engineering Design.

The final publication is available at https://doi.org/10.1007/s00163-018-0301-z
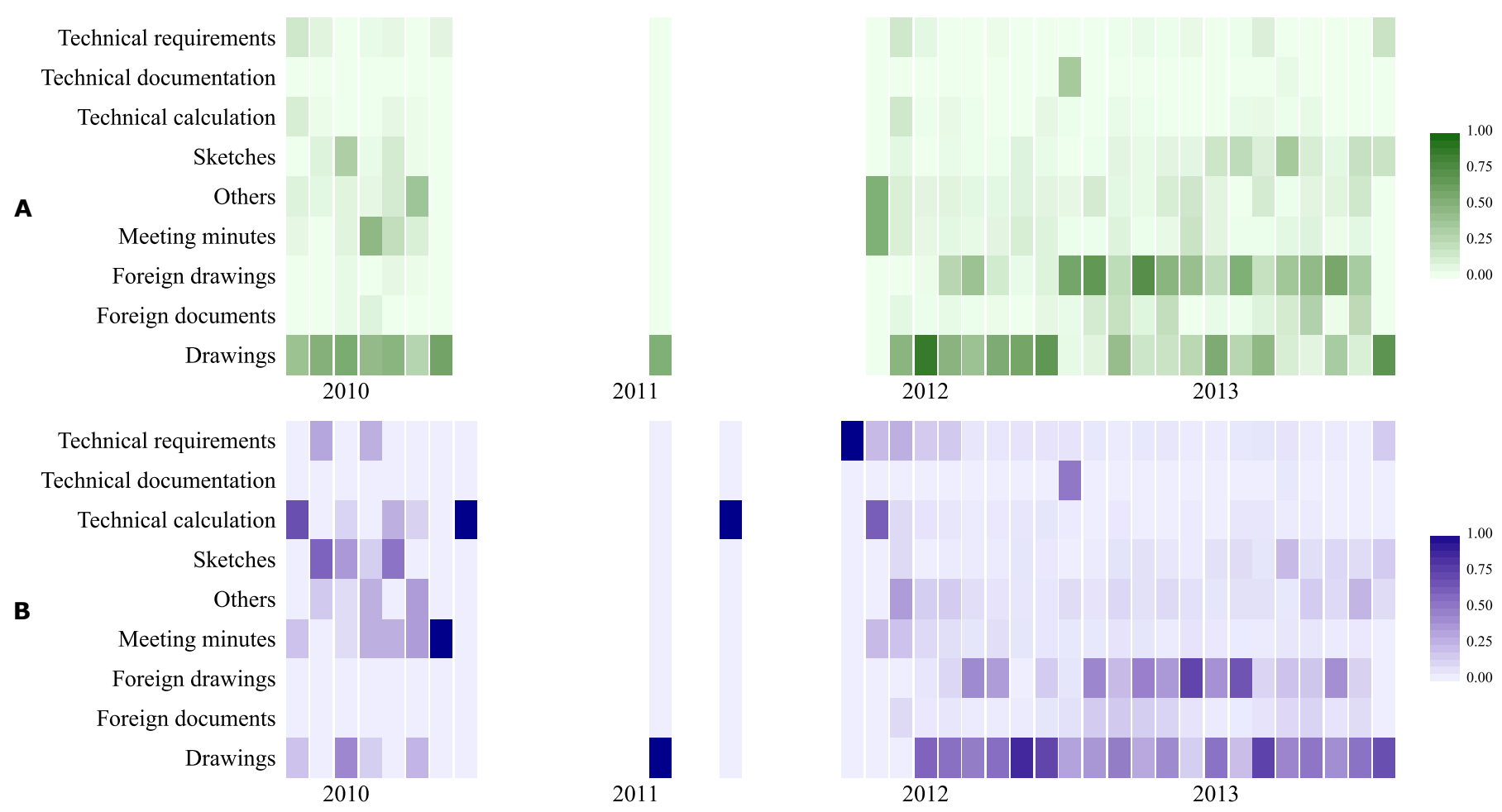

Figure 3: Proportion of type of documents over time. Documents created in green and on top (A) and documents completed in blue and at the bottom (B). The map is read by columns and a column represents one month. White spaces represent no data-logging.

project managers, and decision makers, than our log contains.

Looking at the association between documents and activities, we found that the work during the 'negotiation period' focused mainly on three parts of the power plant: combustion systems, boiler, and pressure parts. From January 2012 onward, we see that technical requirements and technical calculations involve very few new creations or rework. During this period, we observe an increase in relevance of electrical documents and process flow diagrams (category 'Others'). Following the 'negotiation period', we observe that foreign drawings (documents that involve external suppliers) come into play, marking iterations between the company and suppliers. Foreign and internal drawings show alternating dynamics: periods where the focus is more on internal drawings follow periods with more focus on foreign drawings, and vice versa.

We also see a clear 'phase transition' from January 2012. The rhythm of the design process changed and experienced a speeding-up, but also the focus of the work switched. Before January 2012, we observe that people created and completed sketches, specifications, requirements, technical calculations and other conceptual representations. After January 2012, we see that people worked on drawings with the goal of progressing with the design of the biomass power plant. Consistent with this 'phase transition', we observe a shift in the type of iterations: before January 2012, we encounter 'coordination iterations' with the client; after January 2012, we encounter incremental completion as a form of 'progression iterations' 77], with other companies (suppliers). If we were to give those two periods a label, we would say "conceptual design" for the period before January 2012, and "detailed design" for the period after January 2012.

\subsection{Understanding how people influence it- erations during document editing}

Having gained an understanding of how people used the documents in this design process, we can begin to understand how people can influence the document editing process when they interact. In their interactions, designers may iterate documents to communicate and to reach a consensus 9, 10,31. Thus, we conceptualise documents as carriers of information. We use network analysis in order to understand how people can influence the iterations during a document-editing process. To account for the lack of full re- 
This is an author-created postprint version. This work has been published in Research in Engineering Design.

The final publication is available at https://doi.org/10.1007/s00163-018-0301-z

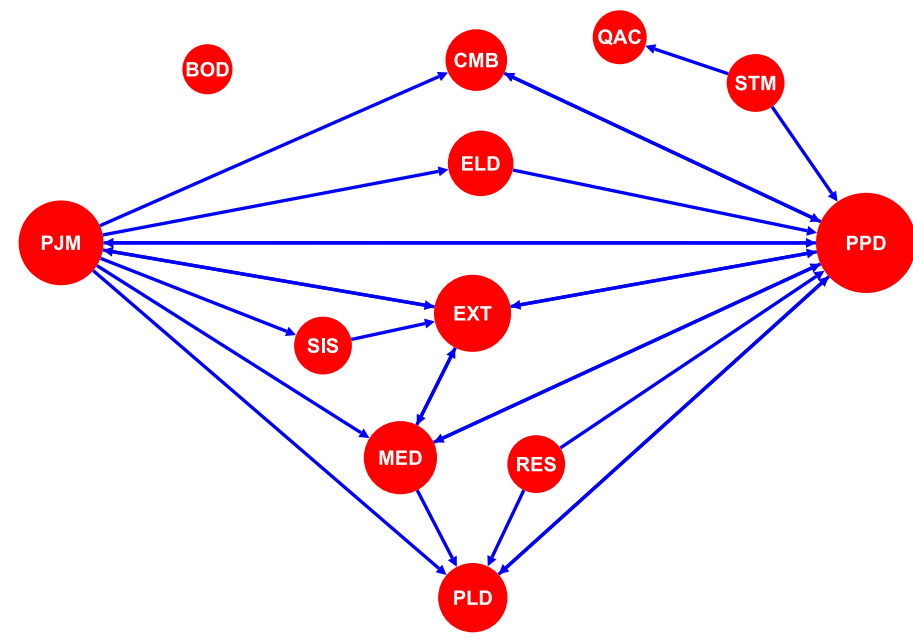

Figure 4: Network between functional units emerging from the document editing process. BOD: Boiler design, PJM: Project management, CMB: Combustion system, ELD: Electrical design, EXT: External consultants, SIS: Site service, MED: Mechanical design, PLD: Plant design, RES: Retrofit service, QAC: Quality assessment, STM: Structural mechanics, PPD: Pressure parts design.

vision history and to understand people in connection with activities and the components of the biomass power plant, we consider relationships between functional units. As each document has a creator and final modifier, we can infer a directed link (information flow) from one functional unit to another based on the proportion of documents they worked on together. In this kind of network, the final modifier has an influence on the creator as the modifier can decide whether the document is satisfactory. If the document is not satisfactory, then it goes back to the creator for new revisions (thus, new iterations).

To understand the influence of the units in the network, we can make a parallel between our document editing network and the structure of trust networks [4] where trust is implicit and represents the fact that each functional unit has to rely on the information produced or processed by other units. With such a network, we could investigate the influence that each functional unit has in this network, in terms of amount of information produced (documents in output) and in terms of amount of information received (documents in input). As such, the term influence does not refer to a form of power or trustworthiness of a given unit, instead it refers to the possibility of a given unit to affect the work of other units in the network through the information generated or processed.

We can have two types of influential nodes: units that facilitate the work because they create many documents that other units complete and units that are authoritative because they review many documents. We could get a first insight about these two roles by simply counting the number of incoming links (to estimate how authoritative a node is) and the number of outgoing links (to estimate how much of a facilitator role a node takes). Such a method, however, would not take into consideration the possible flows that information can follow in the network, giving only a rough estimate of influence from and to direct neighbours and underestimating the possibility that a more peripheral unit could also affect a significant portion of the network. Previous research on trust networks showed that eigenvector-based centrality metrics positively correlate with nodes' degree of trust 4, 20 and appeared better to describe the position of the nodes than other measures.

Thus, given that our network is directed, we can use the algorithm HITS 36 to evaluate how influential people are in this network. HITS provides two centrality measures based on eigenvectors: 'hub', which formalises the intuition that a node is important if it points to many other important nodes (high outgoing flow) and 'authority', which formalises the intuition that a node is important if it is pointed to by many important nodes (high ingoing flow). In our network from the case, such metrics show evidence for the intuition that a functional unit is authoritative in the editing process if it receives documents from many other influential units (as 'authority'), and that a functional unit is a facilitator if it sends documents to many other influential units (as 'hub'). The higher the 'authority' score the more authoritative a unit is and the higher the hub score the more of a 'facilitator' a unit is.

Figure 4 shows a network diagram of the information flows between the functional units. We can see that Boiler design is isolated. This happens because, for all the documents created and completed by this functional unit, both the creator and the final modifier belong to Boiler design. Even though in this design process the boiler is an autonomous sub-system and the functional unit in the company who edit a small amount of documents, this does not mean that Boiler design does not communicate with other functional units. Quality assessment receives documents only from Structural mechanics. Structural mechanics and Retrofit service appear not to receive documents from other functional units, while Site service and Electrical design only receive from Project management. For Site service and Retrofit service, we note that these functional units focus mainly on maintenance and repair. Combustion system receives from both Project management and Pressure parts design.

We can map each functional unit (i.e. team) using their scores of authority and hub (Figure 5) to evaluate their role as 'authority' and 'facilitator'. Retrofit service has a small role as 'facilitator' as they provide documents for Pressure 
This is an author-created postprint version. This work has been published in Research in Engineering Design.

The final publication is available at https://doi.org/10.1007/s00163-018-0301-z

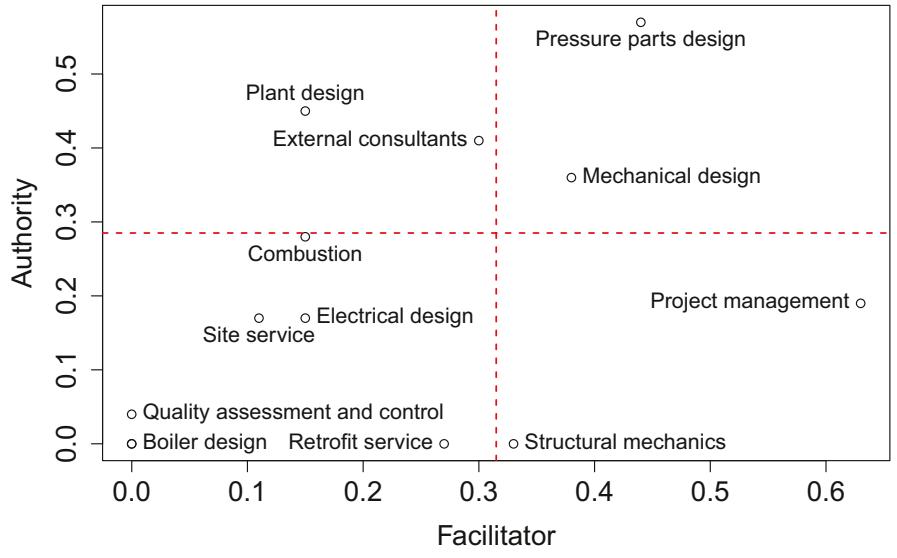

Figure 5: Functional units mapped in their roles of 'authority' and 'facilitator'. The dotted lines that divide the area of the picture into four quadrants are centered on the middle point of each centrality range.

parts design and Plant design. The three functional units that show the highest authority scores are Pressure parts design, Plant design, and External consultants. The three functional units that show the highest facilitator scores are Project management, Pressure part design, and Mechanical design. Project management has a clear role as 'facilitator' as it sends documents to many functional units, especially to 'authorities', which is in line with their coordination and scheduling functions. External consultants have a role as 'authority' as this unit can influence Pressure part design and has a moderate role as 'facilitator' as the unit sends documents to Project management and Mechanical design. This balance between the two roles is in line with the function of consultants hired to solve issues. Mechanical design shows a balance between the roles of 'authority' and 'facilitator', acting as an intermediary between External consultants, Pressure parts design, and Plant design. Plant design has a strong role as 'authority' and a weak role as 'facilitator', which means that it can influence the editing process of other functional units without being able to facilitate the communication in the network. Pressure part design instead shows high scores and thus a strong role as both 'authority' and 'facilitator'.

The network perspective lets us identify two different roles of functional units in relation with iterations. A unit can facilitate iterations and communication by sending information to other units or can act as reviewer when receiving information from other units. Even though the reviewer could increase the number of iterations, its function assures the right information to progress with the design, thus both roles are necessary.

However, we can identify two possibly problematic situa- tions: 1) a unit that has to act as reviewer but not as facilitator can become a 'cul-de-sac' for other units, delaying the progression; 2) a unit that has to act too much both as a reviewer and as facilitator can easily become overloaded by information. The former is the case of 'Plant design', and the latter is the case of 'Pressure parts design'. The second problem can also be extended to the interaction between a unit that has a strong role as authority and a unit that has a strong role as author. We know from previous work that most of the problems happened at interfaces with activities related to pressure parts and external piping (see fig. 4 from 52 ]).

This exploration shows the possible dynamics of design reviews during a design process and how to capture two different roles of the functional units involved in it. It further shows the value of applying network science to better understand certain dynamics of the design process and connects to the theory behind our hypothesis H2, explaining why we expect a positive correlation between the number of revisions and the product of these centrality measures. We have seen how to apply two centrality measures that capture the ingoing and out-going flow for each functional unit and we can now use these centralities in our statistical model to explain the number of iterations $(\sqrt{6})$.

\section{Modelling the iterativeness of the design process through doc- uments}

To test our hypotheses, we develop a multivariate statistical model to fit the number of revisions for each document. Because the number of revisions is a positive integer, the proper strategy to fit a model is to use a regression model suitable for count data. The most natural choice would be a Poisson regression, a type of generalised linear model 49 (GLM) where the random component is specified by the Poisson distribution of the response variable. However, the Poisson regression assumes that the variance be equal to the mean; as we found some overdispersion in our data, this is not the case. There are at least two options to model overdispersed data: the Quasi Poisson regression and the negative binomial regression. The former models the variance to scale linearly with the mean, the latter models the variance to scale quadratically with the mean. After checking the empirical variance-to-mean relationship and finding that a linear scaling is more appropriate, we decided to use the Quasi Poisson regression. The Quasi Poisson regression is a GLM that uses a quasi-likelihood 75 approach where the variance is specified as $\operatorname{var}(x)=\widehat{\Theta \mu}$, where $\Theta$ is the overdispersion index. The result is a model that has the 
This is an author-created postprint version. This work has been published in Research in Engineering Design.

The final publication is available at https://doi.org/10.1007/s00163-018-0301-z

Table 2: Descriptions of the independent variables

\begin{tabular}{ll}
\hline Variable & Description \\
\hline \# Pages & Document's total number of pages \\
Team Size & Size of the team that created the document, in number of people \\
$\log ($ Creation $)$ & Document creation date, as the logarithm of number of days elapsed since the beginning of the project \\
\# Deliverables & Number of document in total produced in the context of the activity with which the document is associated \\
\# Participants & Number of participants/stakeholders involved in the document \\
Facilitator & Prominence in the role of facilitator of the team that created the document \\
Authority & Prominence in the role of authority of the team that created the document \\
Integrative Activity & Binary variable indicating whether the document is associated with an integrative subsystem activity \\
Outdegree & Number of connections in output of the activity with which the document is associated \\
Indegree & Number of connections in input of the activity with which the document is associated \\
$\log ($ Duration $)$ & Document total editing time, in number of days \\
\hline Document type & A set of dummy variables indicating the type of the document (see 4 \\
\hline
\end{tabular}

same estimates as the Poisson regression but more conservative standard errors. With $Y=y_{1}, y_{2}, \ldots y_{n}$ the response variable, the Quasi Poisson regression models each observation $y_{i}$ as drawn from a Poisson distribution with a given mean $y_{i} \sim P\left(\mu_{i}\right)$, where $\mu_{i}$ depends on a set of independent variables $\mathbf{x}_{\mathbf{i}}$. Thus, the model has the following form

$$
\mu_{i}=\exp \left\{\mathbf{x}_{\mathbf{i}}^{\top} \beta\right\}
$$

where $\beta$ is the vector of coefficients. In such a model, the exponentiated regression coefficient $\exp \left\{\beta_{i}\right\}$ represents a multiplicative effect of the i-th coefficient on the mean.

We centered the variables by removing their mean before fitting the models. We fit five models using a strategy called hierarchical regression (not to be confused with hierarchical models). The hierarchical regression consists in fitting a set of nested models to the data to check if the new model represents an improvement over the previous one. All the five models are represented in Table 4. The goodness of the models is evaluated using five measures: the pseudo- $R^{2}$, the Quasi Akaike Information Criterion [5] (QAIC), and the mean squared error (MSE). As it is known that the pseudo$R^{2}$ often increases with the model complexity as i.e. the number of fitted parameters, QAIC is useful because it penalises the quasi-likelihood by the number of parameters used in the model, helping to choose a parsimonious model, where lower scores in QAIC are better. The mean squared error helps us to evaluate the goodness of fit of the model by evaluating the error between the predicted values and the actual ones.

In the following we present the explanatory and control variables that we use to test our hypotheses. We give a brief description of the variables in Table 2 and their correlation along with mean and standard deviation in Table 3

\subsection{Explanatory variables}

We note here that the unit of analysis is the document and the phenomenon of interest is the number of revisions the document received. To test the hypothesis H1, as each document is associated to an activity, we compute in- and outdegree centralities, i.e. the number of in-going and out-going connections from the activity DSM that was developed in previous work 52. We implement the interaction in our model by multiplying in-degree and out-degree (Indegree $\times$ Outdegree).

To test hypothesis $\mathrm{H} 2$, we use the facilitator and authority scores as computed in $\$ 5.3$. We implement the interaction in our model by multiplying facilitator and authority scores (Facilitator $\times$ Authority).

To test hypothesis $\mathrm{H} 3$ we derive the number of participants (\# Participants) for each document from our dataset. Given that each document in our dataset indicates whether the document was sent to the client, to site erection/construction, to quality management, or to the plant quality management, we compressed all such information by counting the number of 'parties' interested in each document. This is consistent with the definitions of stakeholders and participants with decision power previously discussed in the literature review $(\$ 3.3)$.

To test hypothesis $\mathrm{H} 4$, we code a binary variable (Integrative Activity) that indicates if a document is associated with an integrative subsystem activity or not. To this end, we leverage the fact that, by internal policy of the case company, each document is associated with one and only one activity. In previous work [53], a categorisation of activities into modular and integrative was done along with engineers from the case company. We code this binary factor according to that scheme. Thus, documents associated with integrative activities are those documents associated with the following activity groups (see also Table 1): project manage- 
This is an author-created postprint version. This work has been published in Research in Engineering Design.

The final publication is available at https://doi.org/10.1007/s00163-018-0301-z

Table 3: Mean $(\mu)$, standard deviation $(\sigma)$ and correlation between independent variables. In bold, values $>|0.3|$

\begin{tabular}{lrrrrrrrrrrrr}
\hline Variable & $\mu$ & $\sigma$ & 1 & 2 & 3 & 4 & 5 & 6 & 7 & 8 & 9 & 10 \\
\hline 1. \# Pages & 3.4 & 16.5 & & & & & & & & & & \\
2. Team Size & 11.1 & 5.4 & 0.05 & & & & & & & & & \\
3. $\log$ (Creation) & 5.3 & 2.8 & 0.04 & 0.26 & & & & & & & & \\
4. \# Deliverables & 426.5 & 172.1 & -0.16 & -0.10 & -0.19 & & & & & & & \\
5. \# Participants & 0.77 & 0.94 & -0.03 & 0.22 & -0.25 & 0.24 & & & & & & \\
6. Facilitator & 0.34 & 0.15 & 0.01 & $\mathbf{0 . 7 3}$ & 0.21 & -0.09 & 0.12 & & & & & \\
7. Authority & 0.44 & 0.16 & -0.01 & $\mathbf{0 . 8 0}$ & $\mathbf{0 . 3 9}$ & 0.04 & 0.14 & $\mathbf{0 . 6 3}$ & & & & \\
8. Integrative Activity & 0.33 & 0.47 & 0.04 & 0.21 & -0.05 & -0.03 & 0.16 & 0.22 & 0.14 & & & \\
9. Outdegree & 6.54 & 1.96 & 0.14 & 0.22 & 0.05 & -0.23 & 0.00 & 0.23 & 0.12 & 0.24 & & \\
10. Indegree & 6.1 & 1.96 & 0.17 & 0.04 & -0.04 & 0.07 & 0.03 & 0.05 & -0.04 & $\mathbf{0 . 4 9}$ & $\mathbf{0 . 6 3}$ & \\
11. $\log$ (Duration) & 2.66 & 2.1 & -0.06 & 0.06 & -0.11 & 0.12 & $\mathbf{0 . 3 1}$ & 0.02 & -0.03 & 0.09 & 0.08 & 0.07 \\
\hline
\end{tabular}

ment, PFD and P\&ID, steel related activities, COMOS, site erection, and load plan and layout (the last three activity groups are under the label Others in Table 1 .

To test hypothesis H5, we code the document types as a series of binary variables, each of which indicate the type of document discussed in Table 1, checking if foreign documents have higher coefficients than other document types. These dummies are not included in Table 3 for readability reasons, considering that they do not show high correlation with other variables.

To test hypothesis H6, we compute the total editing time for each document as the time elapsed between the creation and the last modification dates, in number of days. We observed that most of the documents are completed within 60 days and that there is a sharp difference in editing time between documents that are not revised, compared to documents that get revisions. For these reasons we implemented the hypothesis by taking the logarithm of the duration $(\log$ (Duration) $)$.

\subsection{Control variables}

Finally, we include the following control variables that could act as possible confounding factors: 1) the number of pages (\# Pages) that the document has, with the intuition that larger documents might be associated with more revisions; 2 ) the $\log$ arithm of the creation date $(\log ($ Creation $))$ as argued in the exploratory data analysis, with the intuition that documents created later on in the process receive fewer revisions; 3 ) the size of the team (Team Size) that produces the document, with the intuition that larger teams editing a higher number of documents might be associated with more revisions than smaller ones; 4) the number of deliverables (\# Deliverables) as the total number of documents produced for each activity, with the intuition that larger activities (in terms of number of documents) might be associated with more revisions than smaller ones.

\section{Results}

We begin our modelling by fitting a model only with the control variables to data (Table 4 Control), observing that this model is a poor fit and that the most explanatory variable is the creation date. If we add the social factors, that is the number of stakeholders with decision power, the centrality measures for the roles of facilitator and authority, their interaction, and their quadratic terms (Table 4 Social), we observe a substantial improvement over the previous one. We note that the interaction term (Facilitator $\times$ Authority) and the number of stakeholders (\# Participants) are significant and positive and in accordance with our hypotheses $\mathrm{H} 3$ and H2. The third model (Table 4 Socio-technical) adds the technical factors, that is the in- and out-degree for the activity network, their interaction, their quadratic terms, and the term for integrative activities. The term for integrative activities (Integrative Activity) and interaction term (Indegree $\times$ Outdegree) are significant and positive, in accordance with hypotheses $\mathrm{H} 5$ and $\mathrm{H} 1$. This model improves the fitting over the previous one, showing that a socio-technical perspective provides a better understanding of iterations than a partial perspective, either social or technical. The full model (Table 4 Full) adds the effects of the design representation (document types) and their editing duration to the previous one. Again, we have an improvement over the previous models and we see that $\log$ (Duration) and document types associated with communication outside the company's boundaries are the only positive coefficients and in accordance with $\mathrm{H} 4$ and $\mathrm{H} 6$. In addition, the coefficients for the other hypotheses are significant and in accordance to our expectations.

Finally, we fit a mixed effects model that adds to the full model random effects to account for unobserved heterogeneity with respect to teams and activities and random effects at the observation level (documents) to account for overdispersion. The coefficients associated with our hypotheses are 
This is an author-created postprint version. This work has been published in Research in Engineering Design.

The final publication is available at https://doi.org/10.1007/s00163-018-0301-z

Table 4: Statistical models. Dependent variable: number of revisions.

\begin{tabular}{|c|c|c|c|c|c|}
\hline & Control & Social & Socio-technical & Full & Mixed effects \\
\hline Intercept & $-0.159(0.036)^{* * *}$ & $-0.328(0.071)^{* * *}$ & $-0.664(0.091)^{* * *}$ & $-1.200(0.156)^{* * *}$ & $-1.614(0.216)^{* * *}$ \\
\hline \multicolumn{6}{|l|}{ Controls: } \\
\hline \# Pages & $-0.003(0.003)$ & $-0.002(0.002)$ & $-0.000(0.002)$ & $0.004(0.001)^{* *}$ & $0.003(0.002)^{*}$ \\
\hline Team Size & $0.090(0.007)^{* * *}$ & $-0.035(0.018)^{*}$ & $-0.127(0.021)^{* * *}$ & $-0.136(0.017)^{* * *}$ & $-0.119(0.037)^{* *}$ \\
\hline $\log ($ Creation $)$ & $-0.146(0.011)^{* * *}$ & $-0.075(0.010)^{* * *}$ & $-0.073(0.011)^{* * *}$ & $-0.196(0.010)^{* * *}$ & $-0.213(0.011)^{* * *}$ \\
\hline \# Deliverables & $-0.001(0.000)^{* * *}$ & $-0.002(0.000)^{* * *}$ & $-0.002(0.000)^{* * *}$ & $-0.002(0.000)^{* * *}$ & $-0.001(0.000)^{* * *}$ \\
\hline \multicolumn{6}{|l|}{ Social factors: } \\
\hline \# Participants & & $0.536(0.029)^{* * *}$ & $0.479(0.032)^{* * *}$ & $0.400(0.028)^{* * *}$ & $0.358(0.031)^{* * *}$ \\
\hline Facilitator & & $4.984(0.707)^{* * *}$ & $6.552(0.772)^{* * *}$ & $6.027(0.661)^{* * *}$ & $5.466(1.294)^{* * *}$ \\
\hline Authority & & $-0.835(0.706)$ & $1.724(0.821)^{*}$ & $1.798(0.652)^{* *}$ & $1.888(1.373)$ \\
\hline Facilitator $^{2}$ & & $-3.600(2.316)$ & $5.153(2.892)$ & $0.783(2.022)$ & $4.544(2.943)$ \\
\hline Authority ${ }^{2}$ & & $-6.903(2.519)^{* *}$ & $-7.002(3.268)^{*}$ & $-7.682(2.081)^{* * *}$ & $-7.382(3.601)^{*}$ \\
\hline Facilitator $\times$ Authority & & $19.968(3.183)^{* * *}$ & $29.201(3.722)^{* * *}$ & $28.022(3.030)^{* * *}$ & $27.204(5.035)^{* * *}$ \\
\hline \multicolumn{6}{|l|}{ Technical factors: } \\
\hline Integrative Activity & & & $0.563(0.075)^{* * *}$ & $0.428(0.059)^{* * *}$ & $0.352(0.061)^{* * *}$ \\
\hline Outdegree & & & $0.193(0.027)^{* * *}$ & $0.142(0.022)^{* * *}$ & $0.119(0.022)^{* * *}$ \\
\hline Indegree & & & $-0.078(0.038)^{*}$ & $-0.058(0.034)$ & $0.022(0.034)$ \\
\hline Outdegree $^{2}$ & & & $-0.026(0.019)$ & $-0.054(0.018)^{* *}$ & $-0.024(0.017)$ \\
\hline Indegree $^{2}$ & & & $-0.084(0.011)^{* * *}$ & $-0.042(0.009)^{* * *}$ & $-0.019(0.009)^{*}$ \\
\hline Outdegree $\times$ Indegree & & & $0.067(0.026)^{*}$ & $0.109(0.024)^{* * *}$ & $0.060(0.023)^{* *}$ \\
\hline \multicolumn{6}{|l|}{ Documents related factors: } \\
\hline $\log$ (Duration) & & & & $0.395(0.015)^{* * *}$ & $0.446(0.016)^{* * *}$ \\
\hline Drawings & & & & $0.073(0.134)$ & $0.321(0.147)^{*}$ \\
\hline Foreign documents & & & & $0.547(0.165)^{* * *}$ & $0.860(0.176)^{* * *}$ \\
\hline Foreign drawings & & & & $0.669(0.134)^{* * *}$ & $1.056(0.156)^{* * *}$ \\
\hline Minutes of Meeting & & & & $-3.844(0.794)^{* * *}$ & $-3.502(0.726)^{* * *}$ \\
\hline Sketches & & & & $0.140(0.298)$ & $0.469(0.290)$ \\
\hline Technical calculation & & & & $-0.150(0.244)$ & $0.085(0.243)$ \\
\hline Technical requirements & & & & $-0.441(0.191)^{*}$ & $-0.202(0.194)$ \\
\hline QAIC & 7349.302 & 6717.211 & 6315.093 & 5174.918 & 5079.545 \\
\hline Pseudo $R^{2}$ & 0.14 & 0.26 & 0.34 & 0.56 & 0.62 \\
\hline MSE & 2.37 & 2.09 & 1.93 & 1.67 & 0.74 \\
\hline Num. obs. & 3088 & 3088 & 3088 & 3088 & 3088 \\
\hline
\end{tabular}

${ }^{* * *} p<0.001,{ }^{* *} p<0.01,{ }^{*} p<0.05$

all significant and in accordance to our expectations. We note that only two of the quadratic terms are now barely significant $(p \geq 0.04)$ while the other two became insignificant. The two barely significant quadratic terms are negative and might suggest an inverted- $\mathcal{U}$. However, a close inspection of the quadratic curves of Authority for varying values of Facilitator and of Indegree for varying values of Outdegree informs us that the maximum of the quadratic functions is often outside the range of values. As such, in our case, the quadratic terms describe monotonic functions rather than inverted- $\mathcal{U}$. As a final check we fitted another model with the same specification as Mixed effects in Table 4 but without the four quadratic terms and performed a likelihood ratio test between the two models. The goodness of fit for the reduced model is not statistically different from the goodness of fit of the model that includes the quadratic terms. Therefore, we can conclude that in our data the quadratic terms for the centralities are irrelevant and were probably capturing part of the heterogeneity now accounted by the random effects. Conversely, the interactions between the centralities are stable and significant across all the models.

We can now empirically answer the proposed hypotheses. Our data support H1 and H2. The interactions, Facilitator $\times$ Authority and Indegree $\times$ Outdegree are positive, indicating a synergistic relation between these centralities and the number of revisions. Indeed, in Figure 6 we plotted the surfaces defined by the couple indegree-outdegree and facilitator-authority. We can see that the number of revisions increases as both indegree and outdegree or, respectively, facilitator and authority increase.

Our data support $\mathrm{H} 3$ as the number of participants is positively associated with the number of revisions: numerically, a unitary increment in the number of participants with de- 


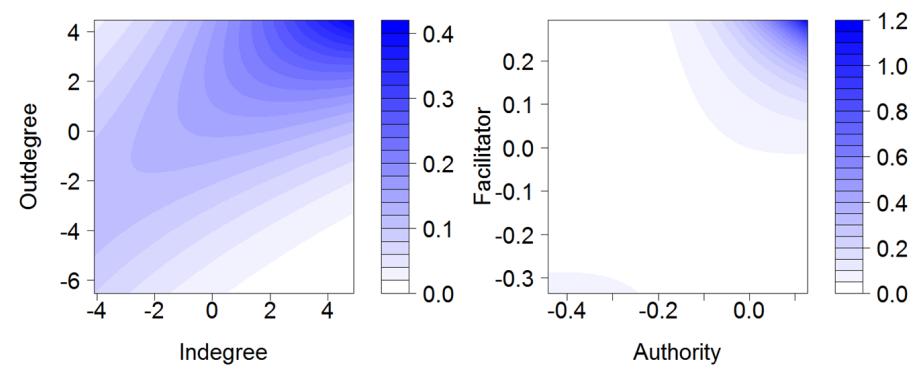

Figure 6: Interactions between indegree and outdegree (left) and authority and facilitator (right). The darker the color the more the iterations. Iterations increase as both centralities increase.

cision power produces, in our model, an increase of $43 \%$ in the number of revisions.

Our data support $\mathrm{H} 4$ as foreign documents and foreign drawings show larger coefficients than other document types. Documents that involve external partners exhibit from $71 \%$ to $100 \%$ more revisions than drawings that do not involve external partners.

Our data support $\mathrm{H} 5$ as documents associated with an integrative activity receive more revisions than documents associated with modular activities. Numerically, a document associated with an integrative activity in our design process is expected to receive $42 \%$ more revisions than a document associated with a modular activity.

Our data support $\mathrm{H} 6$ as documents with a longer editing time receive more revisions. A document with an editing time twice as long as another document, all else equal, is expected to receive $45 \%$ more revisions.

\section{Robustness checks}

We computed the variance inflation factors (VIFs) and observed that they were all smaller than five, thus we do not have multicollinearity issues. Our results are robust to alternative model specifications, such as negative binomial regression or the ordinary least squares regression fitting the log-transformed number of revisions, and to specifications with and without random effects, as already seen in Table 4. The alternative model specifications used as robustness check exhibited, as expected for the reasons discussed in $\$ 6$ that led us to choose the Quasi Poisson regression, lower goodness of fit than the model reported in Table 4 Finally, we refitted our model using 1000 bootstrap replicas and noticed that the bootstrap coefficients and confidence intervals did not change significantly from the coefficients in Table 4 This signifies that our coefficients are not biased and that we do not have extreme observations able to affect the models.
As such, we are confident with respect to the robustness of our results.

\section{Discussion}

Given that the design process is inherently iterative, involving both social and technical factors, this article has sought to explore how these different aspects of the design process influence iterations and how iterations are related to performance. Using data of more than 3000 documents produced during a large-scale design of a biomass power plant, bringing social and technical factors together, showing how they co-exist in a design process and how they can in different and complementary ways shed light on iterations. We show how the combination of social and technical factors can increase the number of iterations. Specifically, we show that structural properties, such as the influence an activity exerts to other activities and its dependence from other activities, are in a synergistic relation with iterations. We find the same result for teams: influence exerted on other teams and the dependence from other teams are in a synergistic relation with iterations. We confirm product modularity as a good design strategy to reduce iterations and suppress change propagation and show how the involvement of external partners and the number of stakeholders/participants interested in a document are positively associated with iterations. Consequently, we provide evidence in favor of an integrated socio-technical perspective to understand engineering design projects and their performance, as such perspective provides a superior understanding. In the following, we discuss our findings, in detail, with respect to the tested hypotheses, their relevance for research and practice, and provide pointers to future work.

\subsection{Completion time and iterations}

In our empirical case, iterations and completion time are positively correlated: documents with higher completion time are associated with more iterations. For instance, a document with completion time of one year receives around two revisions more than a document with completion time of 30 days. This result establishes a link between iterations and execution time and can, in addition, be interpreted as a signature of a different design process model to agile process models where iterations are expected to occur within a range of 2 to 6 weeks 33 . This consideration is confirmed by the creation date, which is negatively associated with iterations, showing that documents created toward the end of this design process receive fewer revisions. This can also be interpreted as a good sign, as reducing the number of iterations toward the end of the process is one of the goals 
This is an author-created postprint version. This work has been published in Research in Engineering Design.

The final publication is available at https://doi.org/10.1007/s00163-018-0301-z

of systems engineering [74].

We also point out that the relation between completion time and iterations does not necessarily need to be one way only. In fact, activities can also be planned to require more execution time because they integrate the output of many activities and provide output for other activities (see also \$3.1) thus being at higher risk of iterations. In terms of planning, this result indicates that an estimation of the completion time for an activity or a document can be a useful indicator of the risk of an activity or a document to be iterated. How to use the estimation of completion time as an early indicator of iteration risk might be an interesting topic for future research.

\subsection{Participants with decision power and iterations}

Coherently with the view of the design process as a social process of negotiations and reaching consensus [9], we showed how our data reflects iterations with the client through the intermittent working patterns shown in Figures 2 and 3 . We go further and show that the higher the number of stakeholders/participants with decision power in the editing process of a document, the higher the number of revisions it will receive. This may be due to the fact that difficulties of coordination and consensus generally increase as the number of parties increases. To support the argument of coordination, we found that documents that involve external partners (in our dataset 'Foreign documents' and 'Foreign drawings') receive more iterations than internal documents, independently of the number of participants. This shows that communication and coordination may be even more demanding and problematic outside the company's boundaries, in analogy with the higher difficulty of cross-department coordination than the difficulty of withindepartment coordination $17,29,65$.

\subsection{Network theory, modularity, and itera- tions}

In this paper, we analysed the relationship between the structure of both process and organisational networks and iterations, under a common framework of nodes' influence and dependence, leveraging the directionality of the edges in the networks. We measured influence and dependence through centrality measures and we found, for both networks, a synergistic relationship between influence and dependence that is positively associated with iterations. As nodes that have both high influence on the network and dependence from others are highly central in the network, they are more likely to be traversed by many flows and be central in error propagation processes $66,54,55,57$. In addition, they are often within cycles in the network and as such, more vulnerable to change propagation [14,67. Our results are fully consistent with this previous work and show that centrality in a network is indeed important and predictive of teams and activities that might be associated with many iterations (through the documents they are connected with). Furthermore, our results show that considering the directionality in networks is important: in accordance with previous work on the effect of component in- and out-hubs on quality $[63$, we found that teams and activities that show high prominence in one between dependence and influence and low prominence in the other are associated with fewer iterations.

Regarding the organisational structure, we focused on the team that produces the document, its ability to influence other teams, captured by the role as authority, and its dependence on other teams, captured by the role as facilitator (see \$5.3). We found that the iterations of the documents produced by a team increase as the role of authority and facilitator of the team co-increase. This finding applies also to the case of interactions between the team that creates the document and the team that reviews it. In such case, the interpretation is that the number of revisions of the document increases as the dependence of the creator team and the influence of the reviewer team increase. Although we did not show this special case in our regression table (Table 4), we also found empirical evidence of this detrimental interaction between two teams that are too prominent in their respective roles. As the centralities take into consideration also the amount of documents processed by each team (thus a form of workload), these findings can straightforwardly be interpreted as an argument for bounded rationality and limited processing capacity $48,59,60$. Indeed, teams that score highly on only one of the two measures, i.e. show high prominence in one between authority and facilitator, produce documents that are associated with fewer revisions (Figure 6). Regarding the process structure, we focused on the focal activity to which the document is connected, its outdegree as a proxy of influence and its indegree as a proxy of dependence. We found that the iterations of the documents produced in the context of a given activity increase as indegree and outdegree co-increase (Figure 6). Again, activities that score highly on only one between indegree and outdegree are associated with fewer revisions. Furthermore, independently from indegree and outdegree, documents connected to integrative subsystem activities, thus referring to integrative components, receive more iterations.

Finally, previous research suggested that component centrality is in an inverted- $\mathcal{U}$ relationship with number of defects [29]. Here, we do not find any evidence supporting this claim and, instead, the quadratic terms have been found 
This is an author-created postprint version. This work has been published in Research in Engineering Design.

The final publication is available at https://doi.org/10.1007/s00163-018-0301-z

not to yield a significant improvement in goodness of fit according to the likelihood ratio test between the model with quadratic terms and the model without quadratic terms. In addition, the shape of the curves is far from an inverted- $\mathcal{U}$. One possible key difference is that we are considering the directionality here while Gokpinar et al. 29 did not. In fact also Sosa et al. 67] considered the directionality of the network and found a monotonic function for the centrality associated with dependence and and a shallow inverted- $\mathcal{U}$ for the centrality associated with influence.

\subsection{Implications for practice}

Results of this study have implications for project and technical managers. For example, in the presence of many stakeholders/participants, common goals and expectations should be defined early on and strategies applied to speed up convergence. More intriguingly, documents could be used to manage certain stakeholders and process execution time for a more speedy achievement of consensus. For example, conceptual representations could be used to iterate ideas and features with the client. Making a parallel with Mihm et al. 47] and their suggestion to keep groups small, we could re-adapt this advice here for documents and suggest keeping the number of stakeholders with decision power connected to a single document low. Insights from network analysis of interactions between teams can be used to reassign workload, reorganise teams, or implement other strategies to improve design reviews. For instance, activities and teams that have both high influence and dependence can be split and the responsibilities reassigned to reduce the overload. An intriguing possibility, in line with $6 \sqrt{63}$, is to strategically use activities that depend on many others but do not influence many other activities as a funnel to integrate dependencies and suppress change propagation for the subsequent activities. Similar reasoning can be applied to teams.

When dealing with integrative subsystem activities, which we showed to be associated with higher number of revisions, technical managers may discuss and define interfaces between modules and teams early in the process, in the attempt to obtain more stable and accurate information. If the interfaces are fixed and well managed, the propagation of errors can be sensibly reduced.

\subsection{Implications for research}

Findings in this research study lead to the following major contributions for research. First, they directly connect the social perspective with design iterations, bringing insights into how the social network of people influences iterations. We have shown how iterations are likely to increase as the number of stakeholders increases and in presence of teams that exhibit high prominence in roles of both facilitator and authority. Thus, we can only envisage further attempts to improve coordination, stakeholder/participant management, and make communication effective and efficient. Second, findings in this paper provide empirical support to the likelihood of iterations happening at integrative subsystem activities and activities with high in-going and out-going information flow. As such, we provide support to previous studies based on modelling and simulations that showed how integrative activities are central to design iterations and their propagation (see for example $[6]$ ). We are not aware of any other studies that provided empirical support for these models and simulations. Such support from real world project data orients scientist toward better and more realistic models for process understanding and performance. Third, continuing the conversation on the importance of document metadata 32 , we show that such metadata is appropriate to gain deep understanding of the design process. From a purely methodological point of view, the analysis presented in this paper could readily and profitably be used by companies to understand where and how to intervene to improve the design process. We used metadata from a real world design process, sourced directly from the company's information management systems. As these systems are not designed with a researcher's goal in mind, we had to make do without the full revision history of the documents. Despite this shortcoming, we have shown a possible use of metadata to study design processes that is focusing on iterations. This study has shown that metadata analysis can be leveraged both to obtain domain knowledge about the design process under analysis and to test scientific hypotheses.

Finally, through our models and linking together process, organisational, and product structure (through the mapping of activities to subsystems), we provide empirical evidence that a joint understanding of these three domains provides superior knowledge of engineering design projects and iterations. We hope our paper will stimulate future research efforts in the socio-technical understanding of engineering projects.

\subsection{Future directions}

We intend to complement this study by obtaining new data with full revision history and possibly document content. Such data may provide even more information to investigate the nature of revisions and shed light on the impact of each revision, and it may also provide important insights about the network formation process. Furthermore, future work may investigate to what extent findings from this case apply to other types of design processes, e.g. original or variant designs. 
This is an author-created postprint version. This work has been published in Research in Engineering Design.

The final publication is available at https://doi.org/10.1007/s00163-018-0301-z

Future research can build on or continue this work in a number of ways. One possibility is to consider the network describing the assignment of people to activities, which has been shown to have important impacts on process robustness 57]. Another possibility is to investigate how people relate to iterations by understanding how they are positioned in different networks related to the process. For instance, by considering the email network and the document team interaction network (considered in this paper) together. This multilayer approach can give more nuanced results as the same people can have different roles in different layers of the network [3]. Understanding of the structural properties that affect iterations may be expanded considering higher order iterations between the centralities and including other factors such as cyclicality 67. Finally, our results suggest strategies to improve process and organisational structures reducing the likelihood of error- and change propagation.

\section{Conclusions}

To show how both social and technical factors influence iterations in a design process, in this study, we analysed metadata of more than 3000 documents that were produced during the design and development of a biomass power plant. We used exploratory data analysis to understand, gain insights, and visualise how the design process unfolds over time, showing its iterativeness. We used a rigorous statistical approach of hypothesis testing to test six hypotheses on what influences design process iterations.

Our findings provide empirical evidence of how the design process can follow a logical evolution despite its inherent iterative nature, suggesting that it is possible to plan process milestones as well as iterations. We find that iterations increase as the number of stakeholders/participants increases, in the presence of integrative activities, in the presence of activities involving external suppliers, in proximity of teams and activities that depend on and can influence overloaded teams, and in proximity of activities that integrate and generate a high amount of information.

We discussed how these findings can readily be used by managers to tackle the challenges arising during the design process and emphasised the relevance of our findings as a platform for future research of design process and project engineering researchers, investigating the interplay between social and technical factors impacting design process performance.

\section{Acknowledgements}

We thank the case company for their continued support. The work reported in this paper is part-funded by the Eu-
roTech Universities Alliance programme, including the Technical University of Denmark and the Technical University of Munich. The exploratory data analysis contained in this paper has been presented at the 21st International Conference on Engineering Design (ICED17) [56].

\section{References}

[1] van der Aalst, W.M.P.: Process Mining: Discovery, Conformance and Enhancement of Business Processes, 1st edn. Springer Publishing Company, Incorporated (2011)

[2] Adler, P.S., Mandelbaum, A., Nguyen, V., Schwerer, E.: From project to process management: An empirically-based framework for analyzing product development time. Management Science 41(3), 458-484 (1995)

[3] Agreste, S., De Meo, P., Ferrara, E., Piccolo, S., Provetti, A.: Analysis of a heterogeneous social network of humans and cultural objects. IEEE Transactions on Systems, Man, and Cybernetics: Systems 45(4), 559$570(2015)$

[4] Agreste, S., De Meo, P., Ferrara, E., Piccolo, S., Provetti, A.: Trust networks: Topology, dynamics, and measurements. IEEE Internet Computing 19(6), 26-35 (2015)

[5] Anderson, D., Burnham, K., White, G.: AIC model selection in overdispersed capture-recapture data. Ecology 75(6), 1780-1793 (1994)

[6] Braha, D., Bar-Yam, Y.: The statistical mechanics of complex product development: Empirical and analytical results. Management Science 53(7), 1127-1145 (2007)

[7] Brody, S.D.: Measuring the effects of stakeholder participation on the quality of local plans based on the principles of collaborative ecosystem management. Journal of Planning Education and Research 22(4), 407-419 (2003). DOI 10.1177/0739456X03022004007

[8] Browning, T.R., Eppinger, S.D.: Modeling impacts of process architecture on cost and schedule risk in product development. IEEE Transactions on Engineering Management 49(4), 428-442 (2002)

[9] Bucciarelli, L.L.: Designing engineers. The MIT Press (1994)

[10] Bucciarelli, L.L.: Between thought and object in engineering design. Design Studies 23(3), 219-231 (2002) 
This is an author-created postprint version. This work has been published in Research in Engineering Design.

The final publication is available at https://doi.org/10.1007/s00163-018-0301-z

[11] Cataldo, M., Herbsleb, J.D., Carley, K.M.: Sociotechnical congruence: a framework for assessing the impact of technical and work dependencies on software development productivity. In: Proceedings of the Second ACM-IEEE international symposium on Empirical software engineering and measurement, pp. 2-11. ACM (2008)

[12] Chen, C.H., Ling, S.F., Chen, W.: Project scheduling for collaborative product development using dsm. International Journal of Project Management 21(4), 291 - 299 (2003). DOI 10.1016/S0263-7863(02)00023-6

[13] Cho, S.H., Eppinger, S.D.: A simulation-based process model for managing complex design projects. IEEE Transactions on Engineering Management 52(3), 316328 (2005). DOI 10.1109/TEM.2005.850722

[14] Clarkson, P.J., Simons, C., Eckert, C.: Predicting change propagation in complex design. Journal of Mechanical Design 126(5), 788-797 (2004)

[15] Cleland, D.I.: Project Stakeholder Management, pp. 275-301. John Wiley \& Sons, Inc. (2008). DOI 10. 1002/9780470172353.ch13

[16] Cockburn, A., Highsmith, J.: Agile software development, the people factor. Computer 34(11), 131-133 (2001)

[17] Colfer, L.J., Baldwin, C.Y.: The mirroring hypothesis: theory, evidence, and exceptions. Industrial and Corporate Change 25(5), 709-738 (2016). DOI $10.1093 / \mathrm{icc} / \mathrm{dtw} 027$

[18] Danese, P., Filippini, R.: Modularity and the impact on new product development time performance: Investigating the moderating effects of supplier involvement and interfunctional integration. International Journal of Operations \& Production Management 30(11), 1191-1209 (2010). DOI 10.1108/01443571011087387

[19] Dawson, J.F.: Moderation in management research: What, why, when, and how. Journal of Business and Psychology 29(1), 1-19 (2014)

[20] De Meo, P., Musial-Gabrys, K., Rosaci, D., Sarnè, G.M., Aroyo, L.: Using centrality measures to predict helpfulness-based reputation in trust networks. ACM Transactions on Internet Technology (TOIT) 17(1), 8 (2017)

[21] Dhillon, B.S.: Engineering design: a modern approach. Richard D Irwin (1996)
[22] Eckert, C., Clarkson, P.J., Zanker, W.: Change and customisation in complex engineering domains. Research in Engineering Design 15(1), 1-21 (2004)

[23] Eppinger, S.D., Browning, T.R.: Design structure matrix methods and applications. The MIT Press (2012)

[24] Eppinger, S.D., Whitney, D.E., Smith, R.P., Gebala, D.A.: A model-based method for organizing tasks in product development. Research in Engineering Design 6(1), 1-13 (1994)

[25] Ford, D.N., Sterman, J.D.: The liar's club: concealing rework in concurrent development. Concurrent Engineering 11(3), 211-219 (2003)

[26] Freeman, R.E.: Strategic management: A stakeholder approach. Cambridge University Press (2010)

[27] Ganzach, Y.: Misleading interaction and curvilinear terms. Psychological Methods 2(3), 235 (1997)

[28] Giffin, M., de Weck, O., Bounova, G., Keller, R., Eckert, C., Clarkson, P.J.: Change propagation analysis in complex technical systems. Journal of Mechanical Design 131(8), 081,001 (2009)

[29] Gokpinar, B., Hopp, W.J., Iravani, S.M.: The impact of misalignment of organizational structure and product architecture on quality in complex product development. Management Science 56(3), 468-484 (2010)

[30] Goldschmidt, G.: Linkography: unfolding the design process. The MIT Press (2014)

[31] Goldschmidt, G., Porter, W. (eds.): Design Representation. Springer (2004)

[32] Gopsill, J., Jones, S., Snider, C., Shi, L., McMahon, C., Hicks, B.: Understanding the engineering design process through the evolution of engineering digital objects. In: 13th International Design Conference, DESIGN 2014. The Design Society (2014)

[33] Highsmith, J., Cockburn, A.: Agile software development: The business of innovation. Computer 34(9), 120-127 (2001)

[34] Hoedemaker, G.M., Blackburn, J.D., Van Wassenhove, L.N.: Limits to concurrency. Decision Sciences 30(1), $1-18$ (1999)

[35] ISO: ISO 9001:2015 quality management systemsrequirements (2015)

[36] Kleinberg, J.M.: Authoritative sources in a hyperlinked environment. Journal of the ACM (JACM) 46(5), 604632 (1999) 
This is an author-created postprint version. This work has been published in Research in Engineering Design.

The final publication is available at https://doi.org/10.1007/s00163-018-0301-z

[37] Kolk, A., Pinkse, J.: Stakeholder mismanagement and corporate social responsibility crises. European Management Journal 24(1), 59 - 72 (2006). DOI http: //dx.doi.org/10.1016/j.emj.2005.12.008

[38] Kusiak, A., Wang, J.: Efficient organizing of design activities. International Journal of Production Research 31(4), 753-769 (1993). DOI 10.1080/ 00207549308956755

[39] Love, P.E., Edwards, D.J., Watson, H., Davis, P.: Rework in civil infrastructure projects: Determination of cost predictors. Journal of Construction Engineering and Management 136(3), 275-282 (2010)

[40] Lyneis, J.M., Ford, D.N.: System dynamics applied to project management: a survey, assessment, and directions for future research. System Dynamics Review 23(2-3), 157-189 (2007)

[41] MacCormack, A., Baldwin, C., Rusnak, J.: Exploring the duality between product and organizational architectures: A test of the mirroring hypothesis. Research Policy 41(8), 1309-1324 (2012)

[42] MacCormack, A., Rusnak, J., Baldwin, C.Y.: Exploring the structure of complex software designs: An empirical study of open source and proprietary code. Management Science 52(7), 1015-1030 (2006)

[43] MacCormack, A., Verganti, R., Iansiti, M.: Developing products on internet time: The anatomy of a flexible development process. Management Science 47(1), 133$150(2001)$

[44] Maier, A.M., Störrle, H.: What are the characteristics of engineering design processes? In: Proceedings of the 18th International Conference on Engineering Design (ICED11), Vol. 1, pp. 188-198. The Design Society (2011)

[45] Meier, C., Yassine, A.A., Browning, T.R.: Design process sequencing with competent genetic algorithms. Journal of Mechanical Design 129(6), 566-585 (2007)

[46] Mihm, J., Loch, C., Huchzermeier, A.: Problemsolving oscillations in complex engineering projects. Management Science 49(6), 733-750 (2003). DOI 10.1287/ mnsc.49.6.733.16021

[47] Mihm, J., Loch, C.H., Wilkinson, D., Huberman, B.A.: Hierarchical structure and search in complex organizations. Management Science 56(5), 831-848 (2010). DOI $10.1287 /$ mnsc. 1100.1148
[48] Miller, G.A.: The magical number seven, plus or minus two: Some limits on our capacity for processing information. Psychological review 63(2), 81 (1956)

[49] Nelder, J., Wedderburn, R.: Generalized linear models. Journal of the Royal Statistical Society. Series A (General) 135(3), 370-384 (1972)

[50] Newcombe, R.: From client to project stakeholders: a stakeholder mapping approach. Construction Management and Economics 21(8), 841-848 (2003). DOI 10.1080/0144619032000072137

[51] Olander, S.: Stakeholder impact analysis in construction project management. Construction Management and Economics 25(3), 277-287 (2007). DOI 10.1080/ 01446190600879125

[52] Parraguez, P., Eppinger, S., Maier, A.: Characterizing design process interfaces as organization networks: Insights for engineering systems management. Systems Engineering 19(2), 158-173 (2016)

[53] Parraguez, P., Eppinger, S.D., Maier, A.M.: Information flow through stages of complex engineering design projects: a dynamic network analysis approach. IEEE Transactions on Engineering Management 62(4), 604$617(2015)$

[54] Pastor-Satorras, R., Vespignani, A.: Epidemic spreading in scale-free networks. Physical review letters 86(14), 3200 (2001)

[55] Pastor-Satorras, R., Vespignani, A.: Immunization of complex networks. Physical Review E 65(3), 036,104 (2002)

[56] Piccolo, S., Jørgensen, S., Maier, A.: Using dataand network science to reveal iterations and phasetransitions in the design process. In: 21st International Conference on Engineering Design (ICED17), vol. 2, pp. 11-20. The Design Society (2017)

[57] Piccolo, S.A., Lehmann, S., Maier, A.: Design process robustness: a bipartite network analysis reveals the central importance of people. Design Science 4, e1 (2018). DOI $10.1017 / \mathrm{dsj} .2017 .32$

[58] Sanchez, R., Mahoney, J.T.: Modularity, flexibility, and knowledge management in product and organization design. Strategic management journal 17(S2), 6376 (1996)

[59] Simon, H.A.: Bounded rationality and organizational learning. Organization Science 2(1), 125-134 (1991). DOI 10.1287/orsc.2.1.125 
This is an author-created postprint version. This work has been published in Research in Engineering Design.

The final publication is available at https://doi.org/10.1007/s00163-018-0301-z

[60] Simon, H.A.: The sciences of the artificial. The MIT Press (1996)

[61] Smith, R.P., Eppinger, S.D.: Identifying controlling features of engineering design iteration. Management Science 43(3), 276-293 (1997)

[62] Smith, R.P., Morrow, J.A.: Product development process modeling. Design Studies 20(3), 237-261 (1999)

[63] Sosa, M., Mihm, J., Browning, T.: Degree distribution and quality in complex engineered systems. Journal of Mechanical Design 133(10), 101,008 (2011)

[64] Sosa, M.E., Eppinger, S.D., Rowles, C.M.: Identifying modular and integrative systems and their impact on design team interactions. Journal of Mechanical Design 125(2), 240-252 (2003)

[65] Sosa, M.E., Eppinger, S.D., Rowles, C.M.: The misalignment of product architecture and organizational structure in complex product development. Management Science 50(12), 1674-1689 (2004). DOI 10.1287/ mnsc. 1040.0289

[66] Sosa, M.E., Eppinger, S.D., Rowles, C.M.: A network approach to define modularity of components in complex products. Journal of Mechanical Design 129(11), 1118-1129 (2007)

[67] Sosa, M.E., Mihm, J., Browning, T.R.: Linking cyclicality and product quality. Manufacturing \& Service Operations Management 15(3), 473-491 (2013)

[68] Steward, D.V.: The design structure system: A method for managing the design of complex systems. IEEE Transactions on Engineering Management EM-28(3), 71-74 (1981)

[69] Terwiesch, C., Loch, C.H., Meyer, A.D.: Exchanging preliminary information in concurrent engineering: Alternative coordination strategies. Organization Science 13(4), 402-419 (2002)

[70] Thomke, S.H.: The role of flexibility in the development of new products: An empirical study. Research Policy 26(1), 105-119 (1997)

[71] Thuesen, C., Hvam, L.: Efficient on-site construction: learning points from a german platform for housing. Construction Innovation 11(3), 338-355 (2011)

[72] Ulrich, K.: The role of product architecture in the manufacturing firm. Research Policy 24(3), 419 - 440 (1995). DOI http://dx.doi.org/10.1016/0048-7333(94) 00775-3
[73] Van Wassenhove, L.N., Besiou, M.: Complex problems with multiple stakeholders: how to bridge the gap between reality and or/ms? Journal of Business Economics 83(1), 87-97 (2013). DOI 10.1007/ s11573-012-0643-3

[74] Walden, D.D., Roedler, G.J., Forsberg, K., Hamelin, R.D., Shortell, T.M.: Systems engineering handbook: A guide for system life cycle processes and activities. Wiley (2015)

[75] Wedderburn, R.W.: Quasi-likelihood functions, generalized linear models, and the Gauss-Newton method. Biometrika 61(3), 439-447 (1974)

[76] Whysall, P.: Stakeholder mismanagement in retailing: A British perspective. Journal of Business Ethics 23(1), 19-28 (2000). DOI 10.1023/A:1006266710132

[77] Wynn, D.C., Eckert, C.M.: Perspectives on iteration in design and development. Research in Engineering Design 28(2), 153-184 (2017)

[78] Yassine, A., Joglekar, N., Braha, D., Eppinger, S., Whitney, D.: Information hiding in product development: The design churn effect. Research in Engineering Design 14(3), 145-161 (2003) 